\title{
Research Article \\ Further Research on the M/G/1 Retrial Queueing Model with Server Breakdowns
}

\author{
Ehmet Kasim and Geni Gupur \\ College of Mathematics and Systems Science, Xinjiang University, Urumqi 830046, China \\ Correspondence should be addressed to Ehmet Kasim, ehmetkasim@163.com
}

Received 13 March 2012; Accepted 28 June 2012

Academic Editor: Song Cen

Copyright (C) 2012 E. Kasim and G. Gupur. This is an open access article distributed under the Creative Commons Attribution License, which permits unrestricted use, distribution, and reproduction in any medium, provided the original work is properly cited.

We study spectral properties of the operator which corresponds to the $M / G / 1$ retrial queueing model with server breakdowns and obtain that all points on the imaginary axis except zero belong to the resolvent set of the operator and 0 is not an eigenvalue of the operator. Our results show that the time-dependent solution of the model is probably strongly asymptotically stable.

\section{Introduction}

There has been considerable interest in retrial queueing systems, see Atencia et al. [1, 2], Choi et al. [3], Djellab [4], Gupur [5, 6], Kasim and Gupur [7], Li et al. [8], Li and Wang [9], Wang et al. [10, 11], and Yang and Templeton [12]. Many researchers studied the M/G/1 retrial queueing systems with server breakdowns in the steady-state case, see Atencia et al. [1, 2], Choi et al. [3], Djellab [4], Li and Wang [9], and Wang et al. [10]. Only few researchers studied transient solutions of M/G/1 retrial queueing systems, see Wang et al. [11], Gupur [5, 6], Kasim and Gupur [7]. And Wang et al. [11] studied the transient solution of the M/G/1 retrial queueing system with server failure by using Laplace transform and obtained the expression of the probability-generating function. In other words, they studied the existence of the time-dependent solution of the model. Gupur [5, 6, 13, 14], Gupur et al. [15], and Kasim and Gupur [7] did dynamic analysis for several queueing models including retrial queueing models by using functional analysis and obtained the existence and uniqueness of the timedependent solution of several queueing models and asymptotic behavior of their timedependent solutions. In this paper, by using the idea in Gupur [6] and Gupur et al. [15], we study the asymptotic behavior of the time-dependent solution of the M/G/1 retrial queueing system with server breakdowns in which the failure states of the server are absorbing states. This queueing system was studied by Wang et al. [10] in 2001. By using 
the supplementary variable technique they established the corresponding queueing model and obtained explicit expressions of some reliability indices such as the availability, failure frequency for steady-state cases under the following hypothesis: "the time-dependent solution of the model converges to a steady-state solution." By reading their paper we find that the above hypothesis, in fact, implies the following two hypotheses.

Hypothesis 1. The model has a nonnegative time-dependent solution.

Hypothesis 2. The time-dependent solution of the model converges to a steady-state solution.

In 2010, Gupur [6] studied the above two hypotheses. Firstly, he converted the model into an abstract Cauchy problem by selecting a suitable Banach space and defining the underlying operator which corresponds to the model and its domain. Next, by using the HilleYosida theorem and the Phillips theorem he proved that the model has a unique non-negative time-dependent solution and therefore obtained that Hypothesis 1 holds. Then, when the service completion rate is a constant, he studied the asymptotic behavior of its timedependent solution. By studying resolvent set of of the adjoint operator of the operator which corresponds to the model (in this case, the M/G/1 retrial queueing model with server breakdowns is called the $M / M / 1$ retrial queueing model with server breakdowns) he obtained the resolvent set of the operator on the imaginary axis: all points on the imaginary axis except zero belong to its resolvent set. And he proved that 0 is not an eigenvalue of the operator. Thus, he suggested that the time-dependent solution of the model probably strongly converges to zero. Until now, any other results about this model have not been found in the literature. In this paper, we try to study the asymptotic behavior of the time-dependent solution of the above model when the service completion rate is a function. Firstly, we convert the model into an abstract Cauchy problem by Gupur [6] and we determine the resolvent set of the adjoint operator of the operator corresponding to the model when the service completion rate satisfies a certain condition. Also, we prove that 0 is not an eigenvalue of the operator. If we can verify 0 is not in the residue spectrum of the operator, then from the above results we conclude that the time-dependent solution of the model strongly converges to zero. Naturally, the results obtained in [6] are now special cases of our results.

According to Wang et al. [10], the M/G/1 retrial queueing system with server breakdowns can be described by the following system of partial differential equations with integral boundary conditions:

$$
\begin{gathered}
\frac{d p_{I, 0,0}(t)}{d t}=-\lambda p_{I, 0,0}(t)+\int_{0}^{\infty} \mu(x) p_{w, 0,1}(x, t) d x, \\
\frac{d p_{I, i, 0}(t)}{d t}=-(\lambda+i \theta) p_{I, i, 0}(t)+\int_{0}^{\infty} \mu(x) p_{w, i, 1}(x, t) d x, \quad i \geq 1, \\
\frac{\partial p_{w, 0,1}(x, t)}{\partial t}+\frac{\partial p_{w, 0,1}(x, t)}{\partial x}=-(\lambda+\alpha+\mu(x)) p_{w, 0,1}(x, t), \\
\frac{\partial p_{w, i, 1}(x, t)}{\partial t}+\frac{\partial p_{w, i, 1}(x, t)}{\partial x}=-(\lambda+\alpha+\mu(x)) p_{w, i, 1}(x, t) \\
+\lambda p_{w, i-1,1}(x, t), \quad i \geq 1, \\
p_{w, i, 1}(0, t)=\lambda p_{I, i, 0}(t)+(i+1) \theta p_{I, i+1,0}(t), \quad i \geq 0, \\
p_{I, 0,0}(0)=1, \quad p_{I, i, 0}(0)=0, \quad i \geq 1 ; \quad p_{w, j, 1}(x, 0)=0, \quad j \geq 0 .
\end{gathered}
$$


Here $(x, t) \in[0, \infty) \times[0, \infty) ; p_{I, i, 0}(t)(i \geq 0)$ represents the probability that the server is idle and there are $i$ customers in the retrial group at time $t ; p_{w, i, 1}(x, t)$ represents the joint probability that at time $t$ there are $i$ customers in the retrial group and the server is up and customer is being served with elapsed service time $x ; \lambda$ is the arrival rate of customers; $\alpha$ is the server failing rate; $\theta$ is the successive interretrial times of customers; $\mu(x)$ is the service completion rate at time $x$ satisfying

$$
\mu(x) \geq 0, \quad \int_{0}^{\infty} \mu(x) d x=\infty .
$$

In this paper, we use the notations in Gupur [6]:

$$
\Gamma=\left(\begin{array}{cccccc}
\lambda & \theta & 0 & 0 & 0 & \ldots \\
0 & \lambda & 2 \theta & 0 & 0 & \ldots \\
0 & 0 & \lambda & 3 \theta & 0 & \ldots \\
0 & 0 & 0 & \lambda & 4 \theta & \ldots \\
\vdots & \vdots & \vdots & \vdots & \vdots & \ddots
\end{array}\right) .
$$

Take a state space as follows:

$$
X=\left\{\begin{array}{l|c}
\left.p_{I}, p_{w}\right) & \begin{array}{c}
\left.p_{I, 0,0}, p_{I, 1,0}, p_{I, 2,0}, \ldots\right) \in l^{1} \\
p_{w}=\left(p_{w, 0,1}, p_{w, 1,1}, p_{w, 2,1}, \ldots\right) \\
\in L^{1}[0, \infty) \times L^{1}[0, \infty) \times L^{1}[0, \infty) \times \cdots \\
\left\|\left(p_{I}, p_{w}\right)\right\|=\sum_{i=0}^{\infty}\left|p_{I, i, 0}\right|+\sum_{i=0}^{\infty}\left\|p_{w, i, 1}\right\|_{L^{1}[0, \infty)}<\infty
\end{array}
\end{array}\right\} .
$$

It is obvious that $X$ is a Banach space and also a Banach lattice. In the following we define operators and their domains:

$$
A\left(p_{I}, p_{w}\right)=\left(\begin{array}{ccccc}
( & \left(\begin{array}{ccccc}
-\lambda & 0 & 0 & 0 & \ldots \\
0 & -(\lambda+\theta) & 0 & 0 & \ldots \\
0 & 0 & -(\lambda+2 \theta) & 0 & \ldots \\
0 & 0 & 0 & -(\lambda+3 \theta) & \ldots \\
\vdots & \vdots & \vdots & \vdots & \ddots
\end{array}\right)\left(\begin{array}{c}
p_{I, 0,0} \\
p_{I, 1,0} \\
p_{I, 2,0} \\
p_{I, 3,0} \\
\vdots
\end{array}\right), \\
& \left(\begin{array}{ccccc}
-\frac{d}{d x} & 0 & 0 & 0 & \ldots \\
0 & -\frac{d}{d x} & 0 & 0 & \ldots \\
0 & 0 & -\frac{d}{d x} & 0 & \ldots \\
0 & 0 & 0 & -\frac{d}{d x} & \ldots \\
\vdots & \vdots & \vdots & \vdots & \ddots
\end{array}\right)\left(\begin{array}{c}
p_{w, 0,1}(x) \\
p_{w, 1,1}(x) \\
p_{w, 2,1}(x) \\
p_{w, 3,1}(x) \\
\vdots
\end{array}\right)
\end{array}\right)
$$




$$
\begin{aligned}
D(A)= & \left\{\begin{array}{c}
\left(p_{I}, p_{w}\right) \in X \\
\end{array} \quad \begin{array}{c}
p_{w, i, 1}(x)(i \geq 0) \text { are absolutely continuous } \\
\text { functions and satisfy } p_{w}(0)=\Gamma p_{I}, \\
\sum_{n=0}^{\infty}\left\|\frac{d p_{w, i, 1}}{d x}\right\|_{L^{1}[0, \infty)}^{<\infty}<
\end{array}\right\}, \\
U\left(p_{I}, p_{w}\right)= & \left(\begin{array}{cccc}
0 & 0 & 0 & \cdots \\
0 & 0 & 0 & \cdots \\
0 & 0 & 0 & \cdots \\
\vdots & \vdots & \vdots & \ddots
\end{array}\right)\left(\begin{array}{c}
p_{I, 0,0} \\
p_{I, 1,0} \\
p_{I, 2,0} \\
\vdots
\end{array}\right), \\
& \left(\begin{array}{cccc}
\mathfrak{u} & 0 & 0 & \cdots \\
\lambda & \mathfrak{u} & 0 & \cdots \\
0 & \lambda & \mathfrak{u} & \cdots \\
\vdots & \vdots & \vdots & \ddots
\end{array}\right)\left(\begin{array}{c}
p_{w, 0,1}(x) \\
p_{w, 1,1}(x) \\
p_{w, 2,1}(x) \\
\vdots
\end{array}\right), D(U)=X,
\end{aligned}
$$

where $\mathfrak{u}=-(\lambda+\alpha+\mu(x))$.

$$
E\left(p_{I}, p_{w}\right)=\left(\left(\begin{array}{c}
\int_{0}^{\infty} \mu(x) p_{w, 0,1}(x) d x \\
\int_{0}^{\infty} \mu(x) p_{w, 1,1}(x) d x \\
\int_{0}^{\infty} \mu(x) p_{w, 2,1}(x) d x \\
\vdots
\end{array}\right),\left(\begin{array}{c}
0 \\
0 \\
0 \\
\vdots
\end{array}\right)\right), \quad D(E)=X
$$

Then the above system of (1.1) can be expressed as an abstract Cauchy problem:

$$
\begin{gathered}
\frac{d\left(p_{I}, p_{w}\right)(t)}{d t}=(A+U+E)\left(p_{I}, p_{w}\right)(t), \quad \forall t \in(0, \infty), \\
\left(p_{I}, p_{w}\right)(0)=\left(\left(\begin{array}{c}
1 \\
0 \\
\vdots
\end{array}\right),\left(\begin{array}{c}
0 \\
0 \\
\vdots
\end{array}\right)\right) .
\end{gathered}
$$

Gupur [6] have obtained the following results.

Theorem 1.1. If $\bar{\mu}=\sup _{x \in[0, \infty)} \mu(x)<\infty$, then $A+U+E$ generates a positive contraction $C_{0}$-semi group $T(t)$. And the system (1.7) has a unique nonnegative time-dependent solution $\left(p_{I}, p_{w}\right)(x, t)=$ $T(t)\left(p_{I}, p_{w}\right)(0)$ satisfying $\left\|\left(p_{I}, p_{w}\right)(\cdot, t)\right\| \leq 1, \forall t \in[0, \infty)$.

\section{Asymptotic Behavior of the Time-Dependent Solution of the System}

In this section, firstly we determine the expression of $(A+U+E)^{*}$, the adjoint operator of $A+U+E$, next we study the resolvent set of $(A+U+E)^{*}$, through which we deduce the resolvent set of $A+U+E$ on the imaginary axis. Thirdly, we prove that 0 is not an eigenvalue of $A+U+E$. Thus, we state our main results in this paper. 
It is easy to see that $X^{*}$, the dual space of $X$, is as follows (see Gupur [6]):

$$
X^{*}=\left\{\begin{array}{c|c}
q_{I}^{*}=\left(q_{I, 0,0}^{*}, q_{I, 1,0}^{*}, q_{I, 2,0}^{*}, \ldots\right) \in l^{\infty}, \\
\left.\left(q_{I}^{*}, q_{w}^{*}\right) \mid \begin{array}{c}
q_{w}^{*}=\left(q_{w, 0,1}^{*}, q_{w, 1,1}^{*}, q_{w, 2,1}^{*}, \ldots\right) \\
\in L^{\infty}[0, \infty) \times L^{\infty}[0, \infty) \times L^{\infty}[0, \infty) \times \cdots \\
\left|\left\|\left(q_{I}^{*}, Q_{w}^{*}\right)\right\|\right|=\sup \left\{\sup _{i \geq 0}\left|q_{I, i, 0}^{*}\right|, \sup _{i \geq 0}\left\|q_{w, i, 1}^{*}\right\|_{L^{\infty}[0, \infty)}\right.
\end{array}\right\}<\infty
\end{array}\right\} .
$$

It is not difficult to verify that $X^{*}$ is a Banach space.

Lemma 2.1. $(A+U+E)^{*}$, the adjoint operator of $A+U+E$, is as follows:

$$
(A+U+E)^{*}\left(q_{I}^{*}, q_{w}^{*}\right)=(\mathcal{L}+\mathcal{G}+\mathscr{L})\left(q_{I}^{*}, q_{w}^{*}\right), \quad \forall\left(q_{I}^{*}, q_{w}^{*}\right) \in D(\mathcal{L}),
$$

where

$$
\begin{aligned}
& \mathcal{L}\left(q_{I}^{*}, q_{w}^{*}\right)=\left(\left(\begin{array}{cccc}
-\lambda & 0 & 0 & \cdots \\
0 & -(\lambda+\theta) & 0 & \cdots \\
0 & 0 & -(\lambda+2 \theta) & \cdots \\
\vdots & \vdots & \vdots & \ddots
\end{array}\right)\left(\begin{array}{c}
q_{I, 0,0}^{*} \\
q_{I, 1,0}^{*} \\
q_{I, 2,0}^{*} \\
\vdots
\end{array}\right)\right. \\
& \left.\left(\begin{array}{ccc}
\frac{d}{d x}-(\lambda+\alpha+\mu(x)) & 0 & \cdots \\
0 & \frac{d}{d x}-(\lambda+\alpha+\mu(x)) & \cdots \\
\vdots & \vdots & \ddots
\end{array}\right)\left(\begin{array}{c}
q_{w, 0,1}^{*}(x) \\
q_{w, 1,1}^{*}(x) \\
\vdots
\end{array}\right)\right) \\
& \mathcal{G}\left(q_{I}^{*}, q_{w}^{*}\right)=\left(\left(\begin{array}{c}
0 \\
0 \\
0 \\
\vdots
\end{array}\right),\left(\begin{array}{cccc}
\mu(x) & 0 & 0 & \cdots \\
0 & \mu(x) & 0 & \cdots \\
0 & 0 & \mu(x) & \cdots \\
\vdots & \vdots & \vdots & \ddots
\end{array}\right)\left(\begin{array}{c}
q_{I, 0,0}^{*} \\
q_{I, 1,0}^{*} \\
q_{I, 2,0}^{*} \\
\vdots
\end{array}\right)\right), \\
& \mathscr{H}\left(q_{I}^{*}, q_{w}^{*}\right)=\left(\left(\begin{array}{ccccc}
\lambda & 0 & 0 & 0 & \cdots \\
\theta & \lambda & 0 & 0 & \cdots \\
0 & 2 \theta & \lambda & 0 & \cdots \\
0 & 0 & 3 \theta & \lambda & \cdots \\
\vdots & \vdots & \vdots & \vdots & \ddots
\end{array}\right)\left(\begin{array}{c}
q_{w, 0,1}^{*}(0) \\
q_{w, 1,1}^{*}(0) \\
q_{w, 2,1}^{*}(0) \\
q_{w, 3,1}^{*}(0) \\
\vdots
\end{array}\right)\right. \\
& \left(\begin{array}{cccccc}
0 & \lambda & 0 & 0 & 0 & \cdots \\
0 & 0 & \lambda & 0 & 0 & \cdots \\
0 & 0 & 0 & \lambda & 0 & \cdots \\
0 & 0 & 0 & 0 & \lambda & \cdots \\
\vdots & \vdots & \vdots & \vdots & \vdots & \ddots
\end{array}\right)\left(\begin{array}{c}
q_{w, 0,1}^{*}(x) \\
q_{w, 1,1}^{*}(x) \\
q_{w, 2,1}^{*}(x) \\
q_{w, 3,1}^{*}(x) \\
\vdots
\end{array}\right),
\end{aligned}
$$




$$
\begin{aligned}
& D(\mathcal{L})=\left\{\begin{array}{l|l}
\left(q_{I}^{*}, q_{w}^{*}\right) \in X^{*} & \begin{array}{c}
q_{w, i, 1}^{*}(x) \text { are absolutely continuous } \\
\text { and satisfy } q_{w, i, 1}^{*}(\infty)=\eta, i \geq 0
\end{array}
\end{array}\right\} \\
& D(\mathcal{G})=D(\mathscr{l})=X^{*} .
\end{aligned}
$$

Here, $\eta$ in $D(\mathcal{L})$ is a nonzero constant which is irrelevant to $i$.

Proof. By using integration by parts and the boundary conditions on $\left(p_{I}, p_{w}\right) \in D(A)$, we have, for any $\left(q_{I}^{*}, q_{w}^{*}\right) \in D(\mathcal{L})$,

$$
\begin{aligned}
& \left\langle(A+U+E)\left(p_{I}, p_{w}\right),\left(q_{I}^{*}, q_{w}^{*}\right)\right\rangle=\sum_{i=0}^{\infty}\left[-(\lambda+i \theta) p_{I, i, 0}+\int_{0}^{\infty} \mu(x) p_{w, i, 1}(x) d x\right] q_{I, i, 0}^{*} \\
& +\int_{0}^{\infty}\left[-\frac{d p_{w, 0,1}(x)}{d x}-(\lambda+\alpha+\mu(x)) p_{w, 0,1}(x)\right] q_{w, 0,1}^{*}(x) d x \\
& +\sum_{i=1}^{\infty} \int_{0}^{\infty}\left[-\frac{d p_{w, i, 1}(x)}{d x}-(\lambda+\alpha+\mu(x)) p_{w, i, 1}(x)+\lambda p_{w, i-1,1}(x)\right] \\
& \times q_{w, i, 1}^{*}(x) d x \\
& =\sum_{i=0}^{\infty}-(\lambda+i \theta) p_{I, i, 0} q_{I, i, 0}^{*}+\sum_{i=0}^{\infty} \int_{0}^{\infty} \mu(x) p_{w, i, 1}(x) q_{I, i, 0}^{*} d x \\
& +\sum_{i=0}^{\infty} \int_{0}^{\infty}-\frac{d p_{w, i, 1}(x)}{d x} q_{w, i, 1}^{*}(x) d x \\
& -\sum_{i=0}^{\infty} \int_{0}^{\infty}(\lambda+\alpha+\mu(x)) p_{w, i, 1}(x) q_{w, i, 1}^{*}(x) d x \\
& +\sum_{i=1}^{\infty} \int_{0}^{\infty} \lambda p_{w, i-1,1}(x) q_{w, i, 1}^{*}(x) d x \\
& =\sum_{i=0}^{\infty}-(\lambda+i \theta) p_{I, i, 0} q_{I, i, 0}^{*}+\sum_{i=0}^{\infty} \int_{0}^{\infty} p_{w, i, 1}(x) \mu(x) q_{I, i, 0}^{*} d x \\
& +\sum_{i=0}^{\infty}\left[\begin{array}{l|l}
-p_{w, i, 1}(x) q_{w, i, 1}^{*}(x) & \begin{array}{l}
x=\infty \\
x=0
\end{array}+\int_{0}^{\infty} p_{w, i, 1}(x) \frac{d q_{w, i, 1}^{*}(x)}{d x} d x
\end{array}\right] \\
& +\sum_{i=0}^{\infty} \int_{0}^{\infty} p_{w, i, 1}(x)\left[-(\lambda+\alpha+\mu(x)) q_{w, i, 1}^{*}(x)\right] d x \\
& +\sum_{i=0}^{\infty} \int_{0}^{\infty} p_{w, i, 1}(x) \lambda q_{w, i+1,1}^{*}(x) d x \\
& =\sum_{i=0}^{\infty}-(\lambda+i \theta) p_{I, i, 0} q_{I, i, 0}^{*}+\sum_{i=0}^{\infty} \int_{0}^{\infty} p_{w, i, 1}(x) \mu(x) q_{I, i, 0}^{*} d x \\
& +\sum_{i=0}^{\infty} p_{w, i, 1}(0) q_{w, i, 1}^{*}(0)
\end{aligned}
$$


Journal of Applied Mathematics

$$
\begin{aligned}
&+\sum_{i=0}^{\infty} \int_{0}^{\infty} p_{w, i, 1}(x)\left[\frac{d q_{w, i, 1}^{*}(x)}{d x}-(\lambda+\alpha+\mu(x)) q_{w, i, 1}^{*}(x)\right] d x \\
&+\sum_{i=0}^{\infty} \int_{0}^{\infty} p_{w, i, 1}(x) \lambda q_{w, i+1,1}^{*}(x) d x \\
&= \sum_{i=0}^{\infty}-(\lambda+i \theta) p_{I, i, 0} q_{I, i, 0}^{*}+\sum_{i=0}^{\infty} \int_{0}^{\infty} p_{w, i, 1}(x) \mu(x) q_{I, i, 0}^{*} d x \\
&+\sum_{i=0}^{\infty}\left[\lambda p_{I, i, 0}+(i+1) \theta p_{I, i+1,0}\right] q_{w, i, 1}^{*}(0) \\
&+\sum_{i=0}^{\infty} \int_{0}^{\infty} p_{w, i, 1}(x)\left[\frac{d q_{w, i, 1}^{*}(x)}{d x}-(\lambda+\alpha+\mu(x)) q_{w, i, 1}^{*}(x)\right] d x \\
&+\sum_{i=0}^{\infty} \int_{0}^{\infty} p_{w, i, 1}(x) \lambda q_{w, i+1,1}^{*}(x) d x \\
&= \sum_{i=0}^{\infty}-(\lambda+i \theta) p_{I, i, 0} q_{I, i, 0}^{*} \\
&+\sum_{i=0}^{\infty} \int_{0}^{\infty} p_{w, i, 1}(x)\left[\frac{d q_{w, i, 1}^{*}(x)}{d x}-(\lambda+\alpha+\mu(x)) q_{w, i, 1}^{*}(x)\right. \\
&+\sum_{i=0}^{\infty} p_{I, i, 0} \lambda q_{w, i, 1}^{*}(0)+\sum_{i=0}^{\infty} p_{I, i+1,0}(i+1) \theta q_{w, i, 1}^{*}(0) \\
&=\left\langle\left(p_{I}, p_{w}\right),(\mathcal{L}+\mathcal{G}+\mathcal{H})\left(q_{I,}^{*}, q_{w}^{*}\right)\right\rangle . \\
&+\lambda
\end{aligned}
$$

From the definition of the adjoint operator and (2.4) we know that the assertion of this lemma is true.

Lemma 2.2. Assume that there exist two positive constant $\bar{\mu}, \mu$ such that

$$
0<\underline{\mu}=\inf _{x \in[0, \infty)} \mu(x) \leq \bar{\mu}=\sup _{x \in[0, \infty)} \mu(x)<\infty .
$$

If $\bar{\mu}<\alpha+\underline{\mu}$, then

$$
\left\{r \in \mathbb{C} \mid \begin{array}{c}
\sup \left\{\frac{\lambda}{|\gamma+\lambda|}, \sup _{i \geq 1}\left\{\frac{\lambda+i \theta}{|\gamma+\lambda+i \theta|}\right\}, \frac{\lambda \bar{\mu}}{|\gamma+\lambda|(\operatorname{Re} \gamma+\lambda+\alpha+\underline{\mu})}+\frac{\lambda}{\operatorname{Re} \gamma+\lambda+\alpha+\underline{\mu}},\right. \\
\left.\sup _{i \geq 1}\left\{\frac{(\lambda+i \theta) \bar{\mu}}{(\operatorname{Re} \gamma+\lambda+\alpha+\underline{\mu})|\gamma+\lambda+i \theta|}+\frac{\lambda}{\operatorname{Re} \gamma+\lambda+\alpha+\underline{\mu}}\right\}\right\}<1, \\
\operatorname{Re} \gamma+\lambda+\alpha+\underline{\mu}>0
\end{array}\right\}
$$


belongs to the resolvent set of $(A+U+E)^{*}$. In particular, all points on the imaginary axis except zero belong to the resolvent set of $(A+U+E)^{*}$, which implies that all points on the imaginary axis except zero belong to the resolvent set of $A+U+E$.

Proof. For any given $\left(y_{I}^{*}, y_{w}^{*}\right) \in X^{*}$, consider the equation $(\gamma I-\mathcal{L}-\mathcal{G})\left(q_{I}^{*}, q_{w}^{*}\right)=\mathscr{H}\left(y_{I}^{*}, y_{w}^{*}\right)$, that is,

$$
\begin{gathered}
(\gamma+\lambda) q_{I, 0,0}^{*}=\lambda y_{w, 0,1}^{*}(0), \\
(\gamma+\lambda+\theta) q_{I, 1,0}^{*}=\theta y_{w, 0,1}^{*}(0)+\lambda y_{w, 1,1}^{*}(0), \\
(\gamma+\lambda+i \theta) q_{I, i, 0}^{*}=i \theta y_{w, i-1,1}^{*}(0)+\lambda y_{w, i, 1}^{*}(0), \quad i \geq 1, \\
\frac{d q_{w, i, 1}^{*}(x)}{d x}=(\gamma+\lambda+\alpha+\mu(x)) q_{w, i, 1}^{*}(x)-\mu(x) q_{I, i, 0}^{*}-\lambda y_{w, i+1,1}^{*}(x), \quad i \geq 0, \\
q_{w, i, 1}^{*}(\infty)=\eta, \quad i \geq 0 .
\end{gathered}
$$

By solving (2.7)-(2.10), we have

$$
\begin{gathered}
q_{I, 0,0}^{*}=\frac{\lambda}{\gamma+\lambda} y_{w, 0,1}^{*}(0), \\
q_{I, i, 0}^{*}=\frac{i \theta}{r+\lambda+i \theta} y_{w, i-1,1}^{*}(0)+\frac{\lambda}{\gamma+\lambda+i \theta} y_{w, i, 1}^{*}(0), \quad i \geq 1,
\end{gathered}
$$

$$
\begin{aligned}
q_{w, i, 1}^{*}(x)= & a_{i} e^{\int_{0}^{x}(\gamma+\lambda+\alpha+\mu(\tau)) d \tau} \\
& +e^{\int_{0}^{x}(\gamma+\lambda+\alpha+\mu(\tau)) d \tau} \int_{0}^{x}\left[-\mu(\tau) q_{I, i, 0}^{*}-\lambda y_{w, i+1,1}^{*}(\tau)\right] e^{-\int_{0}^{\tau}(\gamma+\lambda+\alpha+\mu(s)) d s} d \tau \\
= & a_{i} e^{\int_{0}^{x}(\gamma+\lambda+\alpha+\mu(\tau)) d \tau} \\
& -e^{\int_{0}^{x}(\gamma+\lambda+\alpha+\mu(\tau)) d \tau} \int_{0}^{x}\left[\mu(\tau) q_{I, i, 0}^{*}+\lambda y_{w, i+1,1}^{*}(\tau)\right] e^{-\int_{0}^{\tau}(\gamma+\lambda+\alpha+\mu(s)) d s} d \tau, \quad i \geq 0 .
\end{aligned}
$$

Through multiplying $e^{-\int_{0}^{x}(\gamma+\lambda+\alpha+\mu(\tau)) d \tau}$ to two sides of (2.14), we obtain

$$
e^{-\int_{0}^{x}(\gamma+\lambda+\alpha+\mu(\tau)) d \tau} q_{w, i, 1}^{*}(x)=a_{i}-\int_{0}^{x}\left[\mu(\tau) q_{I, i, 0}^{*}+\lambda y_{w, i+1,1}^{*}(\tau)\right] e^{-\int_{0}^{\tau}(\gamma+\lambda+\alpha+\mu(s)) d s} d \tau, \quad i \geq 0 .
$$

By combining (2.11) with (2.15), we deduce

$$
a_{i}=\int_{0}^{\infty}\left[\mu(\tau) q_{I, i, 0}^{*}+\lambda y_{w, i+1,1}^{*}(\tau)\right] e^{-\int_{0}^{\tau}(\gamma+\lambda+\alpha+\mu(s)) d s} d \tau, \quad i \geq 0 .
$$

By inserting (2.16) into (2.14), we get

$$
q_{w, i, 1}^{*}(x)=e^{\int_{0}^{x}(\gamma+\lambda+\alpha+\mu(\tau)) d \tau} \int_{x}^{\infty}\left[\mu(\tau) q_{I, i, 0}^{*}+\lambda y_{w, i+1,1}^{*}(\tau)\right] e^{-\int_{0}^{\tau}(\gamma+\lambda+\alpha+\mu(s)) d s} d \tau
$$


Journal of Applied Mathematics

9

$$
\begin{aligned}
= & e^{\int_{0}^{x}(\gamma+\lambda+\alpha+\mu(\tau)) d \tau} \int_{x}^{\infty} \mu(\tau) q_{I, i, 0}^{*} e^{-\int_{0}^{\tau}(\gamma+\lambda+\alpha+\mu(s)) d s} d \tau \\
& +\lambda e^{\int_{0}^{x}(\gamma+\lambda+\alpha+\mu(\tau)) d \tau} \int_{x}^{\infty} y_{w, i+1,1}^{*}(\tau) e^{-\int_{0}^{\tau}(\gamma+\lambda+\alpha+\mu(s)) d s} d \tau
\end{aligned}
$$

$$
\begin{aligned}
& \Longrightarrow \\
& \left\|q_{w, i, 1}^{*}\right\|_{L^{\infty}[0, \infty)} \leq \int_{0}^{\infty} e^{\int_{0}^{x}(\operatorname{Re} \gamma+\lambda+\alpha) d \tau} \int_{x}^{\infty} \mu(\tau)\left|q_{I, i, 0}^{*}\right| e^{-\int_{0}^{\tau}(\operatorname{Re} \gamma+\lambda+\alpha) d s} e^{-\int_{x}^{\tau} \mu(s) d s} d \tau d x \\
& +\lambda \int_{0}^{\infty} e^{\int_{0}^{x}(\operatorname{Re} \gamma+\lambda+\alpha) d \tau} \int_{x}^{\infty} \mu(\tau)\left|y_{w, i+1,1}^{*}(\tau)\right| e^{-\int_{0}^{\tau}(\operatorname{Re} \gamma+\lambda+\alpha) d s} e^{-\int_{x}^{\tau} \mu(s) d s} d \tau d x \\
& \leq \frac{\bar{\mu}}{(\operatorname{Re} \gamma+\lambda+\alpha+\underline{\mu})}\left|q_{I, i, 0}^{*}\right| \\
& +\frac{\lambda}{\operatorname{Re} \gamma+\lambda+\alpha+\underline{\mu}}\left\|y_{w, i+1,1}^{*}\right\|_{L^{\infty}[0, \infty)^{\prime}} \quad i \geq 0 .
\end{aligned}
$$

Equation (2.12) gives

$$
\left|q_{I, 0,0}^{*}\right| \leq \frac{\lambda}{|\gamma+\lambda|}\left|y_{w, 0,1}^{*}(0)\right| \leq \frac{\lambda}{|\gamma+\lambda|}\left\|y_{w, 0,1}^{*}\right\|_{L^{\infty}[0, \infty)} .
$$

From (2.13) we estimate

$$
\begin{aligned}
\left|q_{I, i, 0}^{*}\right| & \leq \frac{i \theta}{|\gamma+\lambda+i \theta|}\left|y_{w, i-1,1}^{*}(0)\right|+\frac{\lambda}{|\gamma+\lambda+i \theta|}\left|y_{w, i, 1}^{*}(0)\right| \\
& \leq \frac{i \theta}{|\gamma+\lambda+i \theta|}\left\|y_{w, i-1,1}^{*}\right\|_{L^{\infty}[0, \infty)}+\frac{\lambda}{|\gamma+\lambda+i \theta|}\left\|y_{w, i, 1}^{*}\right\|_{L^{\infty}[0, \infty)} \\
& \leq \frac{\lambda+i \theta}{|\gamma+\lambda+i \theta|} \sup _{i \geq 0}\left\|y_{w, i, 1}^{*}\right\|_{L^{\infty}[0, \infty)^{\prime}} \quad i \geq 1 .
\end{aligned}
$$

By inserting (2.19) and (2.20) into (2.18), we have

$$
\begin{aligned}
\left\|q_{w, 0,1}^{*}\right\|_{L^{\infty}[0, \infty)} \leq & \frac{\bar{\mu}}{(\operatorname{Re} \gamma+\lambda+\alpha+\underline{\mu})} \frac{\lambda}{|\gamma+\lambda|}\left\|y_{w, 0,1}^{*}\right\|_{L^{\infty}[0, \infty)} \\
& +\frac{\lambda}{\operatorname{Re} \gamma+\lambda+\alpha+\underline{\mu}}\left\|y_{w, 1,1}^{*}\right\|_{L^{\infty}[0, \infty)} \\
\leq & \left\{\frac{\lambda \bar{\mu}}{|\gamma+\lambda|(\operatorname{Re} \gamma+\lambda+\alpha+\underline{\mu})}+\frac{\lambda}{\operatorname{Re} \gamma+\lambda+\alpha+\underline{\mu}}\right\}
\end{aligned}
$$




$$
\begin{aligned}
& \times \sup _{i \geq 0}\left\|y_{w, i, 1}^{*}\right\|_{L^{\infty}[0, \infty)^{\prime}} \\
\left\|q_{w, i, 1}^{*}\right\|_{L^{\infty}[0, \infty)} \leq & \frac{\bar{\mu}}{(\operatorname{Re} \gamma+\lambda+\alpha+\underline{\mu})} \frac{\lambda+i \theta}{|\gamma+\lambda+i \theta|} \sup _{i \geq 0}\left\|y_{w, i, 1}^{*}\right\|_{L^{\infty}[0, \infty)} \\
& +\frac{\lambda}{\operatorname{Re} \gamma+\lambda+\alpha+\underline{\mu}} \sup _{i \geq 0}\left\|y_{w, i, 1}^{*}\right\|_{L^{\infty}[0, \infty)}\left[\frac{\lambda}{(\lambda+i \theta) \bar{\mu}}\right. \\
= & \left\{\frac{\underline{\mu}}{(\operatorname{Re} \gamma+\lambda+\alpha+\underline{\mu})|\gamma+\lambda+i \theta|}+\frac{\operatorname{Re} \gamma+\lambda+\alpha+\underline{\mu}}{i \geq 0 .}\right. \\
& \times \sup _{i \geq 0}\left\|y_{w, i, 1}^{*}\right\|_{L^{\infty}[0, \infty)^{\prime}}
\end{aligned}
$$

By combining (2.19) and (2.20) with (2.21), we derive

$$
\begin{aligned}
&\|\|\left(q_{I}^{*}, q_{w}^{*}\right) \| \mid \leq \sup \left\{\frac{\lambda}{|\gamma+\lambda|}, \sup _{i \geq 1}\left\{\frac{\lambda+i \theta}{|\gamma+\lambda+i \theta|}\right\},\right. \\
& \frac{\lambda \bar{\mu}}{|\gamma+\lambda|(\operatorname{Re} \gamma+\lambda+\alpha+\underline{\mu})}+\frac{\lambda}{\operatorname{Re} \gamma+\lambda+\alpha+\underline{\mu}}, \\
&\left.\sup _{i \geq 1}\left\{\frac{(\lambda+i \theta) \bar{\mu}}{(\operatorname{Re} \gamma+\lambda+\alpha+\underline{\mu})|\gamma+\lambda+i \theta|}+\frac{\lambda}{\operatorname{Re} \gamma+\lambda+\alpha+\underline{\mu}}\right\}\right\} .
\end{aligned}
$$

This shows that $\left[I-(\gamma I-\mathcal{L}-\mathcal{G})^{-1} \mathscr{H}\right]^{-1}$ exists and is bounded when $\gamma$ belongs to the set

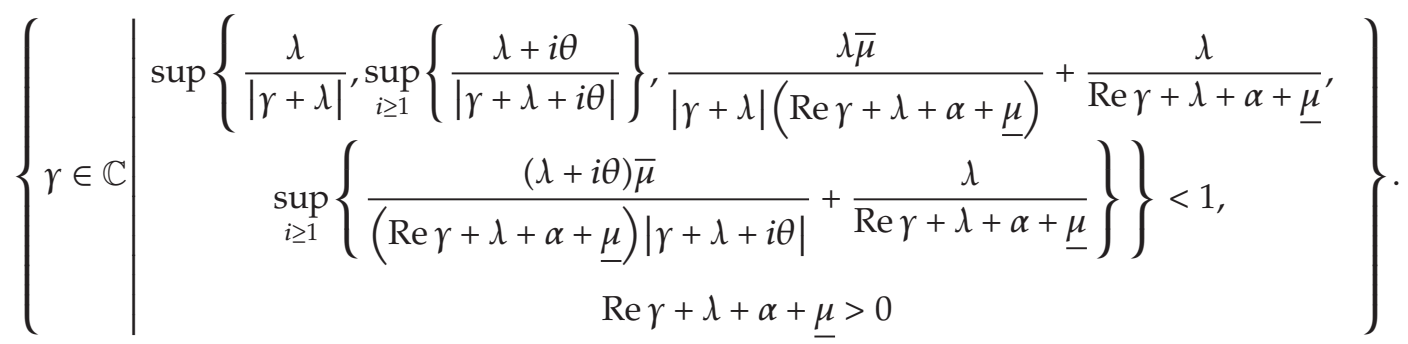

Through discussing the solution of the equation $(\gamma I-\mathcal{L}-\mathcal{G})\left(q_{I}^{*}, q_{w}^{*}\right)=\left(y_{I}^{*}, y_{w}^{*}\right)$ for any given $\left(y_{I}^{*}, y_{w}^{*}\right) \in X^{*}$, it is not difficult to verify $(\gamma I-\mathcal{L}-\mathcal{G})^{-1}$ exists and is bounded when $\gamma$ satisfies 
(2.23). Therefore, by the resolvent equation

$$
\begin{aligned}
{[\gamma I-(\mathcal{L}+\mathcal{G}+\mathscr{H})]^{-1} } & =\left\{(\gamma I-\mathcal{L}-\mathcal{G})\left[I-(\gamma I-\mathcal{L}-\mathcal{G})^{-1} \mathscr{\ell}\right]\right\}^{-1} \\
& =\left[I-(\gamma I-\mathcal{L}-\mathcal{G})^{-1} \mathscr{H}\right]^{-1}(\gamma I-\mathcal{L}-\mathcal{G})^{-1},
\end{aligned}
$$

we know that $(\gamma I-\mathcal{L}-\mathcal{G}-\mathscr{\ell})^{-1}$ exists and is bounded when $\gamma$ belongs to the set (2.23), which means that (2.23) belongs to $\rho(\mathcal{L}+\mathcal{G}+\mathscr{d})$.

In particular, if $\gamma=i a, a \in \mathbb{R} \backslash\{0\}, i^{2}=-1$, then all $\gamma^{\prime}$ s belong to (2.23). In fact, by using the condition on this lemma, we have

$$
\begin{aligned}
& \frac{\lambda}{\sqrt{a^{2}+\lambda^{2}}}<1 \\
& \sup _{k \geq 1} \frac{\lambda+k \theta}{\sqrt{a^{2}+(\lambda+k \theta)^{2}}}<1, \\
& \bar{\mu}<\alpha+\underline{\mu} \Longrightarrow \lambda \bar{\mu}<(\alpha+\underline{\mu}) \sqrt{a^{2}+\lambda^{2}} \\
& \Longrightarrow \lambda \bar{\mu}+\lambda \sqrt{a^{2}+\lambda^{2}}<(\alpha+\underline{\mu}) \sqrt{a^{2}+\lambda^{2}}+\lambda \sqrt{a^{2}+\lambda^{2}} \\
& \Longrightarrow \lambda \bar{\mu}+\lambda \sqrt{a^{2}+\lambda^{2}}<(\lambda+\alpha+\underline{\mu}) \sqrt{a^{2}+\lambda^{2}} \\
& \Longrightarrow \frac{\lambda \bar{\mu}}{\sqrt{a^{2}+\lambda^{2}}(\lambda+\alpha+\underline{\mu})}+\frac{\lambda}{\lambda+\alpha+\underline{\mu}}<1, \\
& \bar{\mu}<\alpha+\underline{\mu} \Longrightarrow(\lambda+k \theta) \bar{\mu}<(\alpha+\underline{\mu}) \sqrt{a^{2}+(\lambda+k \theta)^{2}} \\
& \Longrightarrow(\lambda+k \theta) \bar{\mu}+\lambda \sqrt{a^{2}+(\lambda+k \theta)^{2}}<(\alpha+\underline{\mu}) \sqrt{a^{2}+(\lambda+k \theta)^{2}}+\lambda \sqrt{a^{2}+(\lambda+k \theta)^{2}} \\
& \Longrightarrow(\lambda+k \theta) \bar{\mu}+\lambda \sqrt{a^{2}+(\lambda+k \theta)^{2}}<(\lambda+\alpha+\underline{\mu}) \sqrt{a^{2}+(\lambda+k \theta)^{2}}, \\
& \sup _{k \geq 1}\left\{\frac{(\lambda+k \theta) \bar{\mu}}{\sqrt{a^{2}+(\lambda+k \theta)^{2}}(\lambda+\alpha+\underline{\mu})}+\frac{\lambda}{\lambda+\alpha+\underline{\mu}}\right\}<1 .
\end{aligned}
$$

The above inequalities show that all points on the imaginary axis except zero belong to the resolvent set of $(A+U+E)^{*}$. From the relation between the spectrum of $A+U+E$ and spectrum of $(A+U+E)^{*}$ we know that all points on the imaginary axis except zero belong to the resolvent set of $A+U+E$. 
Lemma 2.3. If

$$
0<\underline{\mu}=\inf _{x \in[0, \infty)} \mu(x) \leq \bar{\mu}=\sup _{x \in[0, \infty)} \mu(x)<\infty,
$$

then 0 is not an eigenvalue of $A+U+E$.

Proof. We consider the equation $(A+U+E)\left(p_{I}, p_{w}\right)=0$, which is equivalent to

$$
\begin{gathered}
\lambda p_{I, 0,0}=\int_{0}^{\infty} \mu(x) p_{w, 0,1}(x) d x, \\
(\lambda+i \theta) p_{I, i, 0}=\int_{0}^{\infty} \mu(x) p_{w, i, 1}(x) d x, \quad i \geq 1, \\
\frac{d p_{w, 0,1}(x)}{d x}=-(\lambda+\alpha+\mu(x)) p_{w, 0,1}(x), \\
\frac{d p_{w, i, 1}(x)}{d x}=-(\lambda+\alpha+\mu(x)) p_{w, i, 1}(x)+\lambda p_{w, i-1,1}(x), \quad i \geq 1, \\
p_{w, i, 1}(0)=\lambda p_{I, i, 0}+(i+1) \theta p_{I, i+1,0}, \quad i \geq 0 .
\end{gathered}
$$

It is hard to determine the concrete expressions of all $p_{I, \mathrm{i}, 0}, p_{w, i, 1}(x)$ and to prove $\left(p_{I}, p_{w}\right) \in$ $D(A)$. In the following we use another method. We introduce the probability-generating functions $Q_{I}(z)=\sum_{i=0}^{\infty} p_{I, i, 0} z^{i}, Q_{w}(x, z)=\sum_{i=0}^{\infty} p_{w, i, 1}(x) z^{i}$ for $|z|<1$. Theorem 1.1 ensures that $Q_{I}(z), Q_{w}(x, z)$ are well-defined. By applying the basic knowledge of power series, the Fubini theorem and (2.27)-(2.28), we have

$$
\begin{gathered}
\lambda p_{I, 0,0}+\sum_{i=1}^{\infty}(\lambda+i \theta) p_{I, i, 0} z^{i}=\int_{0}^{\infty} \mu(x) p_{w, 0,1}(x) d x+\sum_{i=1}^{\infty} \int_{0}^{\infty} \mu(x) p_{w, i, 1}(x) z^{i} d x \\
\Longrightarrow \lambda Q_{I}(z)+\theta z\left(\sum_{i=0}^{\infty} p_{I, i, 0} z^{i}\right)^{\prime}=\int_{0}^{\infty} \mu(x) \sum_{i=0}^{\infty} p_{w, i, 1}(x) z^{i} d x \\
\Longrightarrow \lambda Q_{I}(z)+\theta z \frac{d}{d z} Q_{I}(z)=\int_{0}^{\infty} \mu(x) Q_{w}(x, z) d x .
\end{gathered}
$$

By (2.29) and (2.30), we deduce

$$
\begin{aligned}
\frac{d p_{w, 0,1}(x)}{d x}+\sum_{i=1}^{\infty} \frac{d p_{w, i, 1}(x)}{d x} z^{i}= & -(\lambda+\alpha+\mu(x)) p_{w, 0,1}(x) \\
& -(\lambda+\alpha+\mu(x)) \sum_{i=1}^{\infty} p_{w, i, 1}(x) z^{i}+\sum_{i=1}^{\infty} \lambda p_{w, i-1,1}(x) z^{i} \\
\Longrightarrow & \\
\frac{\partial \sum_{i=0}^{\infty} p_{w, i, 1}(x) z^{i}}{\partial x}= & -(\lambda+\alpha+\mu(x)) \sum_{i=0}^{\infty} p_{w, i, 1}(x) z^{i}+\lambda \sum_{i=1}^{\infty} p_{w, i-1,1}(x) z^{i}
\end{aligned}
$$


Journal of Applied Mathematics

$$
\begin{aligned}
& \Longrightarrow \\
& \frac{\partial Q_{w}(x, z)}{\partial x}=-(\lambda+\alpha+\mu(x)) Q_{w}(x, z)+\lambda z \sum_{i=0}^{\infty} p_{w, i, 1}(x) z^{i} \\
& \Longrightarrow \\
& \frac{\partial Q_{w}(x, z)}{\partial x}=(\lambda z-\lambda-\alpha-\mu(x)) Q_{w}(x, z) \\
& \Longrightarrow \\
& Q_{w}(x, z)=Q_{w}(0, z) e^{\int_{0}^{x}(\lambda z-\lambda-\alpha-\mu(\tau)) d \tau} .
\end{aligned}
$$

Equation (2.31) gives

$$
\begin{aligned}
Q_{w}(0, z) & =\sum_{i=0}^{\infty} p_{w, i, 1}(0) z^{i}=\curlywedge \sum_{i=0}^{\infty} p_{I, i, 0} z^{i}+\sum_{i=0}^{\infty}(i+1) \theta p_{I, i+1,0} z^{i} \\
& =\curlywedge Q_{I}(z)+\theta \frac{d}{d z} Q_{I}(z) .
\end{aligned}
$$

By combining (2.34) with (2.33) and using (2.32), we calculate

$$
\begin{aligned}
\lambda Q_{I}(z)+\theta z \frac{d}{d z} Q_{I}(z) & =\int_{0}^{\infty} \mu(x) Q_{w}(x, z) d x \\
& =\int_{0}^{\infty} \mu(x) Q_{w}(0, z) e^{\int_{0}^{x}[\lambda z-\lambda-\alpha-\mu(\tau)] d \tau} d x \\
& =\left[\lambda Q_{I}(z)+\theta \frac{d}{d z} Q_{I}(z)\right] \int_{0}^{\infty} \mu(x) e^{\int_{0}^{x}[\lambda z-\lambda-\alpha-\mu(\tau)] d \tau} d x \\
& \Longrightarrow \\
& \frac{d Q_{I}(z)}{Q_{I}(z)}=\frac{\lambda}{\theta} \frac{\int_{0}^{\infty} \mu(x) e^{\int_{0}^{x}[\lambda z-\lambda-\alpha-\mu(\tau)] d \tau} d x-1}{z-\int_{0}^{\infty} \mu(x) e^{\int_{0}^{x}[\lambda z-\lambda-\alpha-\mu(\tau)] d \tau} d x} d z \\
& \Longrightarrow \\
& Q_{I}(z)=Q_{I}(0) \exp \left\{\frac{\lambda}{\theta} \int_{0}^{z} \frac{\int_{0}^{\infty} \mu(x) e^{\int_{0}^{x}[\lambda s-\lambda-\alpha-\mu(\tau)] d \tau} d x-1}{s-\int_{0}^{\infty} \mu(x) e^{\int_{0}^{x}[\lambda s-\lambda-\alpha-\mu(\tau)] d \tau} d x} d s\right\} \\
& =p_{I, 0,0} \exp \left\{\frac{\lambda}{\theta} \int_{0}^{z} \frac{\int_{0}^{\infty} \mu(x) e^{\int_{0}^{x}[\lambda s-\lambda-\alpha-\mu(\tau)] d \tau} d x-1}{s-\int_{0}^{\infty} \mu(x) e^{x_{0}^{x}[\lambda s-\lambda-\alpha-\mu(\tau)] d \tau} d x} d s\right\} .
\end{aligned}
$$

This together with (2.34) gives

$$
Q_{w}(0, z)=\frac{\lambda(z-1)}{\left(z-\int_{0}^{\infty} \mu(x) e^{\int_{0}^{x}[\lambda z-\lambda-\alpha-\mu(\tau)] d \tau} d x\right)} Q_{I}(z) .
$$


From (2.35), we derive

$$
\begin{aligned}
\sum_{i=0}^{\infty} p_{I, i, 0} & =\lim _{z \rightarrow 1} Q_{I}(z) \\
& =\lim _{z \rightarrow 1} p_{I, 0,0} \exp \left\{\frac{\lambda}{\theta} \int_{0}^{z} \frac{\int_{0}^{\infty} \mu(x) e^{\int_{0}^{x}[\lambda s-\lambda-\alpha-\mu(\tau)] d \tau} d x-1}{s-\int_{0}^{\infty} \mu(x) e^{\int_{0}^{x}[\lambda s-\lambda-\alpha-\mu(\tau)] d \tau} d x} d s\right\} \\
& =p_{I, 0,0} \exp \left\{\frac{\lambda}{\theta} \int_{0}^{1} \frac{\int_{0}^{\infty} \mu(x) e^{\int_{0}^{x}[\lambda s-\lambda-\alpha-\mu(\tau)] d \tau} d x-1}{s-\int_{0}^{\infty} \mu(x) e^{\int_{0}^{x}[\lambda s-\lambda-\alpha-\mu(\tau)] d \tau} d x} d s\right\}
\end{aligned}
$$

In the following, by the Rouche theorem we know that $s-\int_{0}^{\infty} \mu(x) e^{\int_{0}^{x}[\lambda s-\lambda-\alpha-\mu(\tau)] d \tau} d x$ has a unique zero point inside the unit circle $|z|=1$. And by the residue theorem we determine the above integral.

Let $f(z)=z$ and $g(z)=\int_{0}^{\infty} \mu(x) e^{-(\lambda+\alpha-\lambda z) x-\int_{0}^{x} \mu(\tau) d \tau} d x$. It is well known that $f(z)$ and $g(z)$ are differentiable inside and continuous on the contour $|z|=1$. And $|f(z)|=1$ on $|z|=1$. Since, for $(\lambda+\alpha-\lambda \operatorname{Re} z)>0$,

$$
\begin{aligned}
|g(z)|= & \left|\int_{0}^{\infty} \mu(x) e^{-(\lambda+\alpha-\lambda z) x-\int_{0}^{x} \mu(\tau) d \tau} d x\right| \\
\leq & -\int_{0}^{\infty} e^{-(\lambda+\alpha-\lambda \operatorname{Re} z) x} d e^{-\int_{0}^{x} \mu(\tau) d \tau} \\
= & -\left[e^{-(\lambda+\alpha-\lambda \operatorname{Re} z) x} e^{-\int_{0}^{x} \mu(\tau) d \tau} \mid \begin{array}{c}
x=\infty \\
x=0
\end{array}\right. \\
& \left.+(\lambda+\alpha-\lambda \operatorname{Re} z) \int_{0}^{\infty} e^{-(\lambda+\alpha-\lambda \operatorname{Re} z) x-\int_{0}^{x} \mu(\tau) d \tau} d x\right] \\
= & 1-(\lambda+\alpha-\lambda \operatorname{Re} z) \int_{0}^{\infty} e^{-(\lambda+\alpha-\lambda \operatorname{Re} z) x-\int_{0}^{x} \mu(\tau) d \tau} d x \\
< & 1=|f(z)|,
\end{aligned}
$$

we have $|f(z)|<|g(z)|$ on $|z|=1$. Consequently, all conditions of Rouche's theorem are satisfied. Obviously $f(z)-g(z)$ has only one zero inside the unit circle $|z|=1$, since $f(z)$ has one. If we denote this zero by $z_{0}$, then it is a simple pole of

$$
\frac{\int_{0}^{\infty} \mu(x) e^{-(\lambda+\alpha-\lambda z) x-\int_{0}^{x} \mu(\tau) d \tau} d x-1}{z-\int_{0}^{\infty} \mu(x) e^{-(\lambda+\alpha-\lambda z) x-\int_{0}^{x} \mu(\tau) d \tau} d x} .
$$


By the residue theorem, we calculate

$$
\begin{gathered}
\int_{0}^{1} \frac{\int_{0}^{\infty} \mu(x) e^{-(\lambda+\alpha-\lambda s) x-\int_{0}^{x} \mu(\tau) d \tau} d x-1}{s-\int_{0}^{\infty} \mu(x) e^{-(\lambda+\alpha-\lambda s) x-\int_{0}^{x} \mu(\tau) d \tau} d x} d s \\
\quad=\lim _{z \rightarrow z_{0}} \frac{\int_{0}^{\infty} \mu(x) e^{-(\lambda+\alpha-\lambda z) x-\int_{0}^{x} \mu(\tau) d \tau} d x-1}{1-\int_{0}^{\infty} \lambda x \mu(x) e^{-(\lambda+\alpha-\lambda z) x-\int_{0}^{x} \mu(\tau) d \tau} d x} \\
\quad=\frac{\int_{0}^{\infty} \mu(x) e^{-\left(\lambda+\alpha-\lambda z_{0}\right) x-\int_{0}^{x} \mu(\tau) d \tau} d x-1}{1-\int_{0}^{\infty} \lambda x \mu(x) e^{-\left(\lambda+\alpha-\lambda z_{0}\right) x-\int_{0}^{x} \mu(\tau) d \tau} d x} .
\end{gathered}
$$

Thus, we obtain

$$
\sum_{i=0}^{\infty} p_{I, i, 0}=p_{I, 0,0} \exp \left\{\frac{\lambda}{\theta} \frac{\int_{0}^{\infty} \mu(x) e^{-\left(\lambda+\alpha-\lambda z_{0}\right) x-\int_{0}^{x} \mu(\tau) d \tau} d x-1}{1-\int_{0}^{\infty} \lambda x \mu(x) e^{-\left(\lambda+\alpha-\lambda z_{0}\right) x-\int_{0}^{x} \mu(\tau) d \tau} d x}\right\}<\infty
$$

From (2.36), we derive

$$
\begin{aligned}
\lim _{z \rightarrow 1} Q_{w}(0, z) & =\lim _{z \rightarrow 1} \frac{\lambda(z-1)}{z-\int_{0}^{\infty} \mu(x) e^{\int_{0}^{x}[\lambda z-\lambda-\alpha-\mu(\tau)] d \tau} d x} Q_{I}(z) \\
& =\lim _{z \rightarrow 1} \frac{\lambda(z-1)}{z-\int_{0}^{\infty} \mu(x) e^{\int_{0}^{x}[\lambda z-\lambda-\alpha-\mu(\tau)] d \tau} d x} \lim _{z \rightarrow 1} Q_{I}(z) \\
& =\frac{\lambda(1-1)}{1-\int_{0}^{\infty} \mu(x) e^{-\int_{0}^{x}[\alpha+\mu(\tau)] d \tau} d x} Q_{I}(1) \\
& =0 .
\end{aligned}
$$

By combining (2.33) with (2.42), we estimate

$$
\begin{aligned}
\sum_{i=0}^{\infty} p_{w, i, 1}(x) & =\lim _{z \rightarrow 1} Q_{w}(x, z)=\lim _{z \rightarrow 1} Q_{w}(0, z) e^{\int_{0}^{x}(\lambda z-\lambda-\alpha-\mu(\tau)) d \tau} \\
& =\lim _{z \rightarrow 1} Q_{w}(0, z) e^{-\int_{0}^{x}(\alpha+\mu(\tau)) d \tau} \\
& =0 .
\end{aligned}
$$

Since Theorem 1.1 shows that each $p_{w, i, 1}(x)$ is nonnegative, (2.43) implies $p_{w, i, 1}(x)=0$ for all $i \geq 0$. This together with (2.27) and (2.28) gives $p_{I, i, 0}=0$ for all $i \geq 0$. In other words, $(A+U+E)\left(p_{I}, p_{w}\right)=0$ has only zero solution; that is, 0 is not an eigenvalue of $A+U+E$. 
Lemma 2.3 shows that the system (1.7) does not have nonzero steady-state solution.

If we can prove that 0 is not in the residue spectrum of $A+U+E$, then by Theorem 1.1, Lemmas 2.2 and 2.3, and ABLV Theorem (see [16] or [17]), we deduce that the timedependent solution of system (1.7) is strongly asymptotically stable. This result is quite different from other queueing models, see Gupur [13-15] and Kasim and Gupur [7].

\section{Acknowledgments}

The authors' research work is supported by Science Foundation of Xinjiang University (no: XY110101) and the Natural Science Foundation of Xinjiang (no: 2012211A023).

\section{References}

[1] I. Atencia, G. Bouza, and P. Moreno, "An $M^{[X]} / G / 1$ retrial queue with server breakdowns and constant rate of repeated attempts," Annals of Operations Research, vol. 157, pp. 225-243, 2008.

[2] I. Atencia, I. Fortes, P. Moreno, and S. Sánchez, "An $M / G / 1$ retrial queue with active breakdowns and Bernoulli schedule in the server," International Journal of Information and Management Sciences, vol. 17 , no. 1, pp. 1-17, 2006.

[3] B. D. Choi, K. B. Choi, and Y. W. Lee, " $M / G / 1$ retrial queueing systems with two types of calls and finite capacity," Queueing Systems, vol. 19, no. 1-2, pp. 215-229, 1995.

[4] N. V. Djellab, "On the single-server retrial queue," Yugoslav Journal of Operations Research, vol. 16, no. 1 , pp. 45-63, 2006.

[5] G. Gupur, "Semigroup method for $M / G / 1$ retrial queue with general retrial times," International Journal of Pure and Applied Mathematics, vol. 18, no. 4, pp. 405-429, 2005.

[6] G. Gupur, "Analysis of the $M / G / 1$ retrial queueing model with server breakdowns," Journal of Pseudo-Differential Operators and Applications, vol. 1, no. 3, pp. 313-340, 2010.

[7] E. Kasim and G. Gupur, "Spectral properties of the $M^{[X]} / G / 1$ operator and its application," Journal of Pseudo-Differential Operators and Applications, vol. 2, no. 2, pp. 219-287, 2011.

[8] Q.-L. Li, Y. Ying, and Y. Q. Zhao, "A BMAP/G/1 retrial queue with a server subject to breakdowns and repairs," Annals of Operations Research, vol. 141, pp. 233-270, 2006.

[9] J. Li and J. Wang, "An $M / G / 1$ retrial queue with second multi-optional service, feedback and unreliable server," Applied Mathematics. A Journal of Chinese Universities. Ser. B, vol. 21, no. 3, pp. 252$262,2006$.

[10] J. Wang, J. Cao, and Q. Li, "Reliability analysis of the retrial queue with server breakdowns and repairs," Queueing Systems, vol. 38, no. 4, pp. 363-380, 2001.

[11] J. Wang, B. Liu, and J. Li, "Transient analysis of an $M / G / 1$ retrial queue subject to disasters and server failures," European Journal of Operational Research, vol. 189, no. 3, pp. 1118-1132, 2008.

[12] T. Yang and J. G. C. Templeton, "A survey of retrial queues," Queueing Systems. Theory and Applications, vol. 2, no. 3, pp. 201-233, 1987.

[13] G. Gupur, "Advances in queueing models' research," Acta Analysis Functionalis Applicata, vol. 13, no. 3, pp. 225-245, 2011.

[14] G. Gupur, "Time-dependent analysis for a queue modeled by an infinite system of partial differential equations," Science China, vol. 55, no. 5, pp. 985-1004, 2012.

[15] G. Gupur, X. Li, and G. Zhu, Functional Analysis Method in Queueing Theory, Research Information, Herdfortshire, UK, 2001.

[16] W. Arendt and C. J. K. Batty, "Tauberian theorems and stability of one-parameter semigroups," Transactions of the American Mathematical Society, vol. 306, no. 2, pp. 837-852, 1988.

[17] Yu. I. Lyubich and V. Q. Phóng, "Asymptotic stability of linear differential equations in Banach spaces," Polska Akademia Nauk. Instytut Matematyczny, vol. 88, no. 1, pp. 37-42, 1988. 


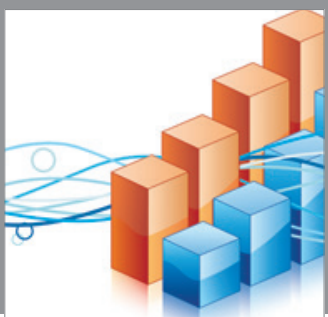

Advances in

Operations Research

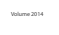

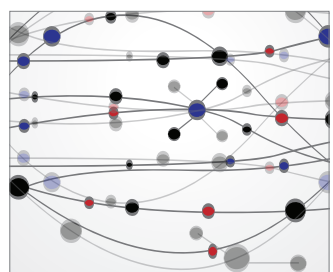

\section{The Scientific} World Journal
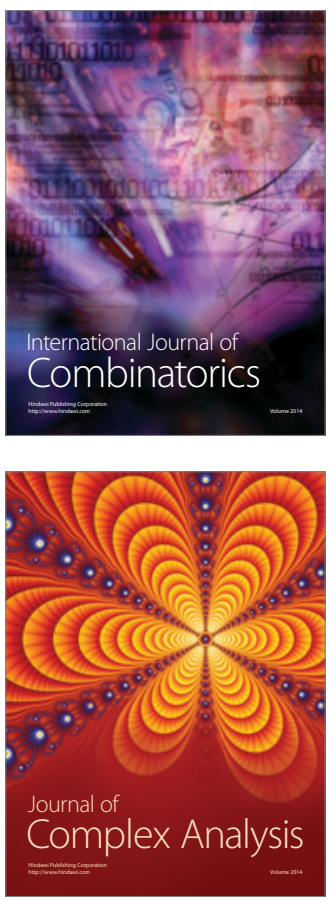

International Journal of

Mathematics and

Mathematical

Sciences
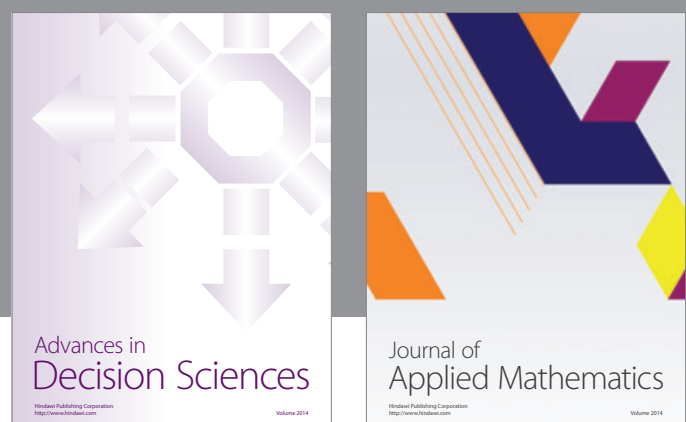

Journal of

Applied Mathematics
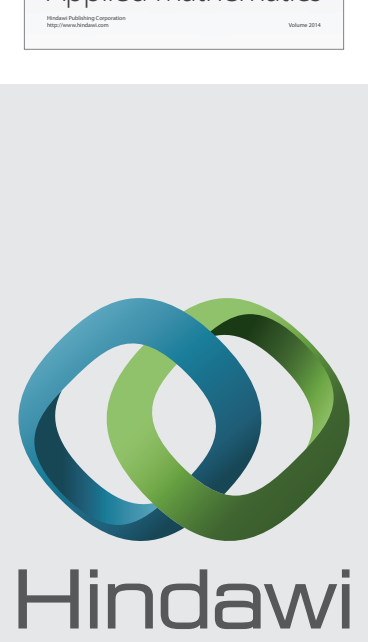

Submit your manuscripts at http://www.hindawi.com
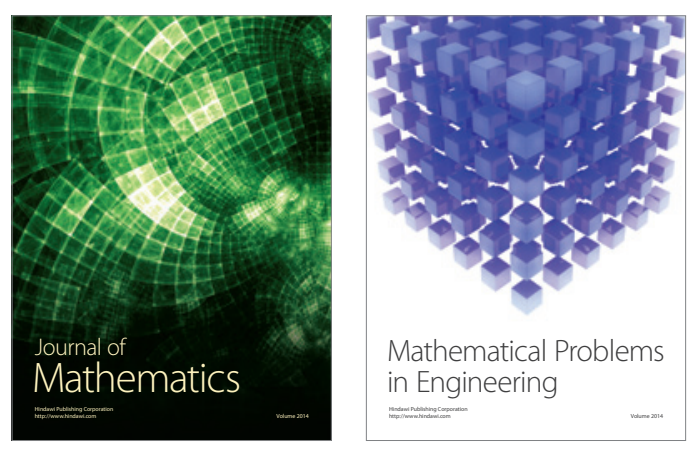

Mathematical Problems in Engineering
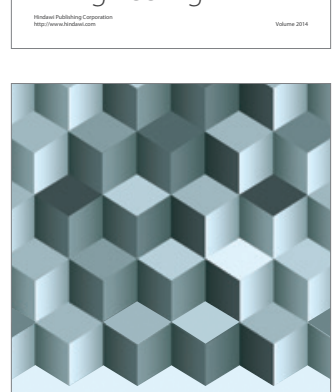

Journal of

Function Spaces
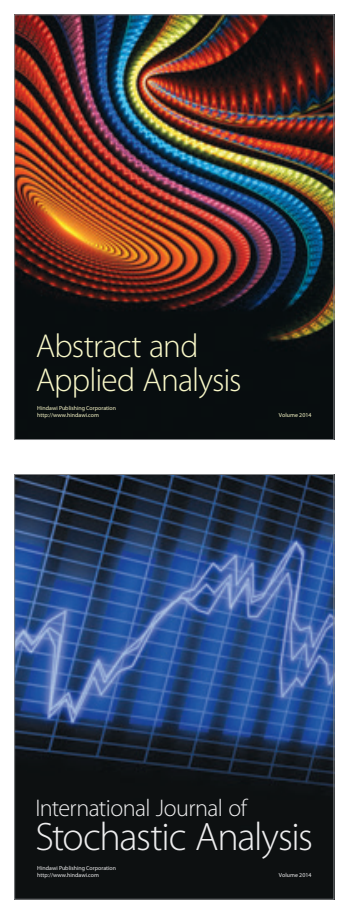

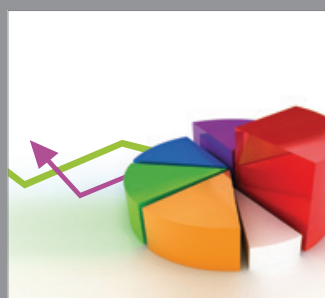

ournal of

Probability and Statistics

Promensencen
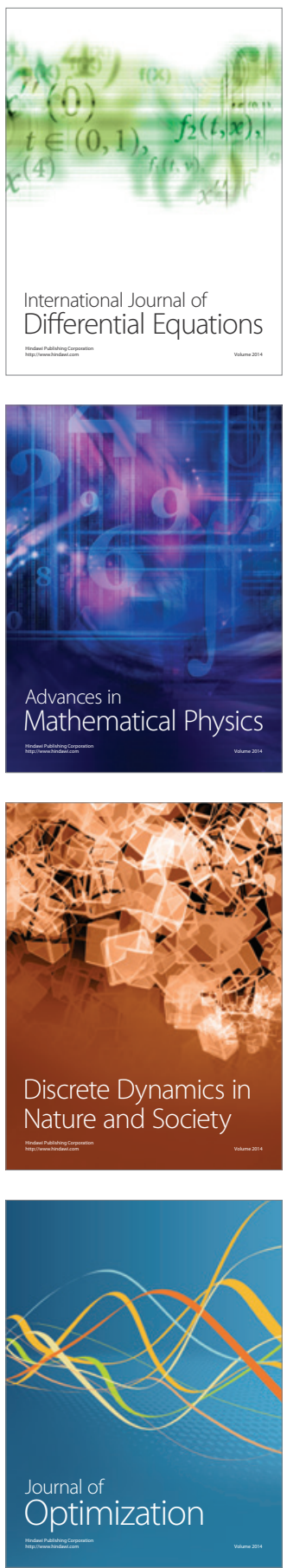